\title{
ABSTRACT \\ JWST Lightweight Mirror TRL-6 Results
}

\author{
H. Philip Stahl \\ NASA MSFC
}

Mirror technology for a Primary Mirror Segment Assembly (PMSA) is a system of components: reflective coating; polished optical surface; mirror substrate; actuators, mechanisms and flexures; and reaction structure. The functional purpose of a PMSA is to survive launch, deploy and align itself to form a 25 square meter collecting area 6.5 meter diameter primary mirror with a $131 \mathrm{~nm}$ rms wavefront error at temperatures $<50 \mathrm{~K}$ and provide stable optical performance for the anticipated thermal environment. At the inception of JWST in 1996, such a capability was at a Technology Readiness Level (TRL) of 3. A highly successful technology development program was initiated including the Sub-scale Beryllium Mirror Demonstrator (SBMD) and Advanced Mirror System Demonstrator (AMSD) projects. These projects along with flight program activities have matured mirror technology for JWST to TRL-6. A directly traceable prototype (and in some cases the flight hardware itself) has been built, tested and operated in a relevant environment. 


\title{
JWST Lightweight Mirror TRL-6 Results
}

\author{
H. Philip Stahl \\ NASA MSFC XD-30 \\ Huntsville, AL 35812 \\ (256) $544-0445$ \\ h.philip.stanl@nasa.gov
}

\begin{abstract}
Mirror technology for a Primary Mirror Segment Assembly (PMSA) is a system of components: reflective coating; polished optical surface; mirror substrate; actuators, mechanisms and flexures; and reaction structure. The functional purpose of a PMSA is to survive launch, deploy and align itself to form a 25 square meter collecting area 6.5 meter diameter primary mirror with a $131 \mathrm{~nm} \mathrm{rms}$ wavefront error at temperatures $<50 \mathrm{~K}$ and provide stable optical performance for the anticipated thermal environment. At the inception of JWST in 1996, such a capability was at a Technology Readiness Level (TRL) of 3. A highly successful technology development program was initiated including the Sub-scale Beryllium Mirror Demonstrator (SBMD) and Advanced Mirror System Demonstrator (AMSD) projects. These projects along with flight program activities have matured mirror technology for JWST to TRL-6. A directly traceable prototype (and in some cases the flight hardware itself) has been built, tested and operated in a relevant environment.
\end{abstract}

\section{TABLE OF CONTENTS}

\section{INTRODUCTION \\ 2. MIRROR TECHNOLOGY \\ 3. TRL-6 COMPLIANCE \\ 4. SUMMARY \\ REFERENCES \\ BIOGRAPHY}

\section{INTRODUCTION}

Since the initial Design Studies leading to JWST (Dressler, 1996; Stockman 1997), Mirror Technology has been identified as a (if not the) critical capability necessary to enable the next generation of large aperture space telescopes required to achieve the science goals of imaging the earliest galaxies and proto-galaxies after the big bang. Specific telescope architectures were explored via three independent design concept studies conducted during the summer of 1996 (Coulter, 1998). Achieving the desired science objectives required a never before demonstrated space telescope capability, one with an 8 meter class primary mirror that is diffraction limited at 2 micrometers and operating in deep space at temperatures well below $70 \mathrm{~K}$. (NGST Monograph No 1, 1999) Beryllium was identified

\footnotetext{
1 "U.S. Government work not protected by U.S. copyright."

${ }^{2}$ IEEEAC paper \#1186, Version 2, Updated 18 December 2006.
}

in the NASA "Yardstick" design as the preferred material because of its ability to provide stable optical performance in the anticipated thermal environment as well as its excellent specific stiffness.

Because of launch vehicle constraints, two very significant architectural constraints were placed upon the telescope: segmentation and areal density. Each of these directly resulted in specific technology capability requirements. First, because the maximum launch vehicle payload fairing diameter is approximately 4.5 meters, the only way to launch an 8 meter class mirror is to segment it, fold it and deploy it on orbit - resulting in actuation and control requirements. Second, because of launch vehicle mass limits, the primary mirror allocation was only $1000 \mathrm{~kg}-$ resulting in a maximum areal density specification of 20 $\mathrm{kg} / \mathrm{m} 2$. (Bely, 1999) Overcoming these specific architectural constraints while achieving the required imaging quality (all within a cost constrained programmatic environment) was the primary focus of the three 1996 concept studies conducted by Lockheed-Martin, TRW and NASA GSFC, and the subsequent Pre-Phase A Architecture Studies conducted by TRW and Ball Aerospace.

An assessment of the pre-1996 state of the art indicated that the necessary mirror technology (as demonstrated by existing space, ground and laboratory test bed telescopes) was at a Technology Readiness Level 3. (Table 1) The largest space telescope was Hubble's 2.4 meter glass mirror which operates at $300 \mathrm{~K}$ and has an areal density of 180 $\mathrm{kg} / \mathrm{m} 2$. Ground telescopes such as Keck and Hobby-Eberly had demonstrated 10 meter class semi-actively controlled segmented mirrors. But, as ground telescopes they are exceedingly massive (2000 $\mathrm{kg} / \mathrm{m} 2)$ and thermally unsuitable. Test beds such as the Itek Large Optical Telescope (ALOT) (Cox, 1996) and the Kodak Advanced Optical System Demonstrator (AOSD) had demonstrated proof of concept for 4 meter class pseudo-space-qualifiable actively-controlled segmented telescopes in a laboratory environment. And the US Air Force Large Active Mirror Project (LAMP) had demonstrated a 4 meter class actively controlled segmented primary mirror operating in a vacuum environment (although at $300 \mathrm{~K}$ ). But again, these test beds were $2 \mathrm{X}$ to $6 \mathrm{X}$ too massive for JWST ( 50 to $150 \mathrm{~kg} / \mathrm{m} 2$ ) and thermally unsuitable. Finally, the largest cryogenic mirror under development was the 0.85 meter diameter Infrared Telescope Technology Testbed (ITTT) beryllium primary mirror which would eventually fly in the Spitzer Space Telescope in 2003. 


\begin{tabular}{|l|c|c|c|c|c|l|}
\hline \multicolumn{7}{|c|}{ Table 1. 1996 JWST Optical System Requirements State of Art } \\
\hline Parameter & JWST & Hubble & Spitzer & Keck & LAMP & Units \\
\hline Aperture & 8 & 2.4 & 0.85 & 10 & 4 & meters \\
\hline Segmented & Yes & No & No & 36 & 7 & Segments \\
\hline Areal Density & 20 & 180 & 28 & 2000 & 140 & kg/m2 \\
\hline Diffraction Limit & 2 & 0.5 & 6.5 & 10 & Classified & micrometers \\
\hline Operating Temp & $<50$ & 300 & 5 & 300 & 300 & K \\
\hline Environment & L2 & LEO & Drift & Ground & Vacuum & Environment \\
\hline Substrate & TBD & ULE Glass & I-70 Be & Zerodur & Zerodur & Material \\
\hline Architecture & TBD & Passive & Passive & Hexapod & Adaptive & Control \\
\hline First Light & TBD & 1993 & 2003 & 1992 & 1996 & First Light \\
\hline
\end{tabular}

\section{MIRROR TECHNOLOGY}

Based on the state of art assessment and the three architectural concept studies, it was concluded that the JWST mission was feasible - provided that a well planned, aggressive technology development effort was implemented early in the development phase. (Coulter, 1998) The JWST Project initiated a systematic mirror technology development program. The goal of the effort was to advance mirror design and associated manufacturing process technologies that would dramatically reduce the cost, schedule and weight for large-aperture space optical systems. A critical element of the program was competition. Competition between ideas and vendors resulted in a remarkably rapid TRL advance in the state of the art for large-aperture lightweight cryogenic space mirrors. Furthermore, it resulted in significant reductions in the manufacturing cost and schedule. The mirror technology development effort successfully reduced technical (weight \& performance) and programmatic (schedule \& cost) risks by fabricating full-scale mirror systems and validating their performance under flight-like operational conditions.

The mirror technology development program explored key elements necessary to produce TRL-6 mirrors for JWST, including: Substrate Material (glass, beryllium, silicon carbide, nickel, etc; mechanical, thermal and optical material properties; ability to manufacture large enough substrates; etc); Mirror Design (open back, closed back, arched, thin face sheet; launch loads; etc); Architecture (passive, active, rigid, semi-rigid, etc); Fabrication Process (substrate fabrication, grind \& polish, coating); Metrology (vibration insensitivity, cryogenic characterization, etc) and Performance (cryogenic, thermal, mechanical, launch loads, etc). (Smith, 2001; Stahl, 2001) Full and sub-scale mirror systems and their constituent components (i.e. flexures, coatings and actuators) were fabricated and cryogenically tested. Significant investments were made in facilities, equipment, procedures and expertise. Also, to improve the ability of models to accurately predict on-orbit performance, an extensive program was conducted to characterize the cryogenic properties (i.e. CTE and CTE uniformity, dynamic dampening, stiffness and tensile strength) of various mirror and structure materials as well as their susceptibility to micrometeoroid impacts.
Given that large lightweight mirrors are critical to multiple government missions and that their fabrication poses significant technical challenges which directly translate into programmatic cost and schedule risks, NASA was joined by several Department of Defense (DoD) partners. An excess of $\$ 40 \mathrm{M}$ was invested in mirror technology development from 1998 to 2004 . This investment occurred through a series of related contracts managed by Marshall Space Flight Center as the Lead for Mirror Technology Development including: SBMD (Sub-scale Beryllium Mirror Demonstrator), NMSD (NGST Mirror System Demonstrator), AMSD (Advanced Mirror System Demonstrator) and several other small technology studies and SBIR (Small Business Innovative Research) contracts. Additional mirror technology development activities were conducted under two Pre-Phase A Architecture Study Contracts awarded to TRW and Lockheed.

SBMD (Subscale Beryllium Mirror Demonstrator) (Figure 1) was a contract with Ball Aerospace to produce a $0.53-\mathrm{m}$ diameter beryllium mirror with a 20 meter radius of curvature mounted on a solid Be support structure. (Reed, 2001) It was cryo-tested multiple times and provided invaluable experience and learning which was applied to AMSD. Additionally, some JWST technologies were matured to TRL-6 via SBMD.

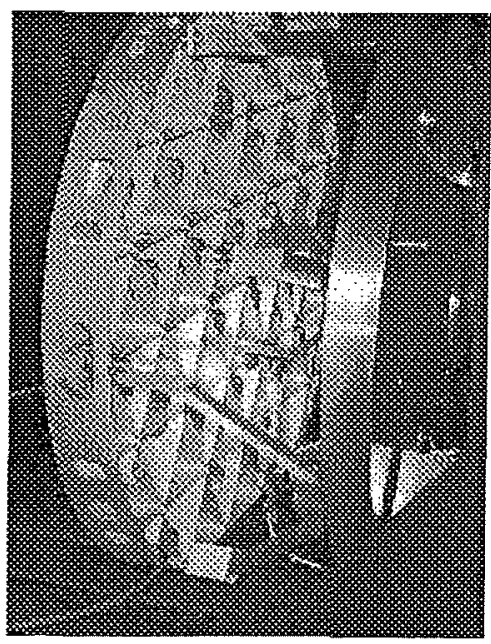

Figure $1 \mathrm{SBMD}$ 
AMSD (Advanced Mirror System Demonstrator) was a joint NASA and DOD program. While some mission requirements were divergent, the pooling of resources provided greater funding to explore the technology landscape more widely and deeply. AMSD followed a phased down-select approach. Phase 1 awarded contracts to five different vendors to study and develop designs for a total of eight different mirror architectures. The best three of these designs were then funded for fabrication in Phase 2. Ball Aerospace, Goodrich and Kodak were the winning vendors. All of these mirrors were 1.3 to 1.4 meters in diameter - just the size needed to produce a segmented mirror six to eight meters in diameter - and have an areal density of approximately $15 \mathrm{~kg} / \mathrm{m} 2$. Ball, building upon their earlier SBMD work, developed a beryllium mirror that incorporates radius of curvature (RoC) and mirror position control utilizing flight-like cryogenic actuators. (Figure 2)

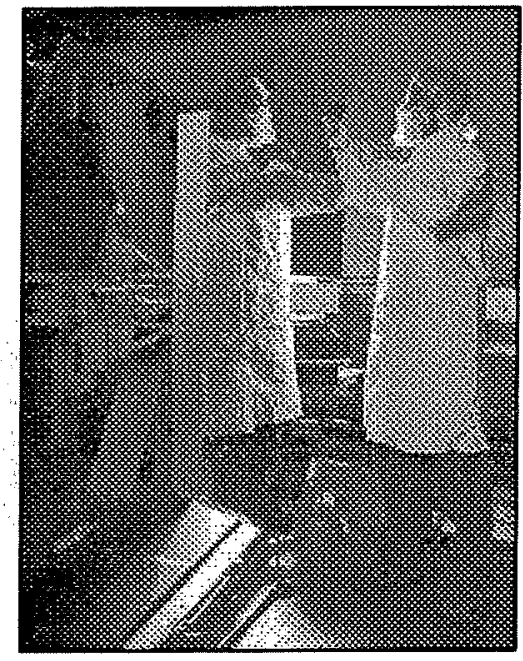

Figure 2 Ball AMSD

Goodrich developed a high-authority mirror consisting of a shallow-ribbed glass facesheet supported on an array of displacement actuators. Kodak pursued a semi-rigid mirror architecture that utilized a very lightweight all-glass cellular-core mirror along with a few force actuators to correct low-order mirror distortions that arise on cool down to cryogenic temperatures. After the selection of Northrop Grumman as JWST prime contractor, the Goodrich effort was terminated due to compatibility issues with the Northrop Grumman JWST architecture. This allowed more focused funding on the remaining Ball and Kodak mirrors. Both mirrors were successful cryogenic tested to characterize their cryogenic performance. Upon the completion of AMSD Phase II, a JWST OTE Optical Readiness (OOR) review was held which compared the readiness of the Ball and Kodak mirror technology for implementation into the Northrop Grumman Optical Telescope Element (OTE) architecture. The mirrors were compared on multiple factors including performance, cost, schedule and risk. The Ball beryllium mirror was selected for flight. (JWST-RVW-002463; Stahl, 2004)

\section{TRL-6 COMPLIANCE}

\section{Requirements Flowdown}

Primary Mirror Segment Assembly (PMSA) Technology Requirements are fully traceable from Level 1 Science Requirements through Level 2 Mission Requirements and Level 3 Observatory Requirements. (Table 2) Level 1 science requirements are defined in the JWST Program Plan, (Rev A; 2006). Level 2 mission requirements are defined in the JWST Mission Requirements Document (Rev N, 2006).

Level 2 requirements were significantly refined and clarified during the Pre-Phase A Architecture Study (TRW, 2001). Level 3 observatory requirements and specific mirror technology component requirements were derived during the Phase 2 NGST Observatory Contract and refined once the Prime Contractor (and Implementation Team) was selected. Complete PMSA requirements are defined in the Equipment Specification for JWST PMSA (Ball, 2005)

\begin{tabular}{|c|c|c|}
\hline \multicolumn{3}{|c|}{ Table 2 PMSA Requirement Traceability } \\
\hline Level 1 Requirements & Level 2 Requirements & PMSA Technology \\
\hline \multirow[t]{2}{*}{ L1-01: Science Spectral Range } & \multirow[t]{2}{*}{ MR-211: Optical Transmission } & PMSA-110: Spectral Reflectance 0.6-27 micrometers \\
\hline & & PMSA-530: Operational Temperature $28-50 \mathrm{~K}$ \\
\hline L1-04: Celestial C & MR-115: EE Stability & PMSA-170: Surface Fig Thermal Change $<0.3 \mathrm{~nm} \mathrm{rms} / \mathrm{K}$ \\
\hline \multirow{2}{*}{ L1-12: L2 Orbit } & MR-099: Mass & PMSA-410: Mass $<39.17 \mathrm{~kg}$ (Areal Density $<26.5 \mathrm{~kg} / \mathrm{m} 2)$ \\
\hline & MR-283: Launch Vehicle & PMSA-180: Surface Distortion from Launch $<2.9 \mathrm{~nm} \mathrm{rms}$ \\
\hline L1-13: PM Collecting Area & MR-198: PM Collecting Area & PMSA-70: Polished Surface Area $>1.46 \mathrm{sq} \mathrm{m}(1.3 \mathrm{~m}$ dia $)$ \\
\hline \multirow[t]{4}{*}{ L1-14: Observatory Strehl Ratio } & \multirow[t]{4}{*}{ MR-228: OTE WFE } & PMSA-150: Uncorrectable Surface Error $<23.7 \mathrm{~nm} \mathrm{rms}$ \\
\hline & & PMSA-195: Surface Change from Creep $<1.8 \mathrm{nmrms}$ \\
\hline & & PMSA-1560: ROC Adjustment Resolution $<10 \mathrm{~nm}$ pv sag \\
\hline & & PMSA-370: Hexapod 6 DOF (Piston Resolution $<10 \mathrm{~nm}$ ) \\
\hline L1-16: Thermal Environment & MR-122: Thermal Emission & PMSA-530: Operational Temperature $28-50 \mathrm{~K}$ \\
\hline
\end{tabular}

A comparison of these PMSA requirements with the preJWST state of the art for space telescopes, as defined by Hubble and Spitzer, (Table 3) clearly shows that they were truly well beyond the state of the art (SOA. Thus, these capabilities are TRL-6 technologies which must be demonstrated. 


\begin{tabular}{|l|l|c|c|}
\hline \multicolumn{4}{|c|}{ Table 3 JWST Mirror Technology vs State of Art } \\
\hline \multicolumn{1}{|c|}{ PMSA Technology } & JWST Requirement & Hubble & Spitzer \\
\hline PMSA-110: Spectral Reflectance 0.6-28 $\mu \mathrm{m}$ & $\begin{array}{l}\text { Gold Coating on O-30 Be } \\
\text { with 28K Survival }\end{array}$ & UV/Visible & Uncoated \\
\hline PMSA-530: Operational Temperature 28-50K & $<7.5 \mathrm{~nm}$ rms for 30 to 55K & & \\
\hline PMSA-170: Surface Figure Thermal Change & Areal Density $<26.5 \mathrm{~kg} / \mathrm{m} 2$ & $180 \mathrm{~kg} / \mathrm{m} 2$ & $28 \mathrm{~kg} / \mathrm{m} 2$ \\
\hline PMSA-410: Mass < 39.17 kg & $<2.9 \mathrm{~nm} \mathrm{rms}$ & & $<\sim 20 \mathrm{~nm} \mathrm{~mm}$ \\
\hline PMSA-180: Surface Distortion from Launch & 1.3 meter diameter Segment & $2.4 \mathrm{~meter}$ & $0.85 \mathrm{~meter}$ \\
\hline PMSA-70: Polished Surface Area & $<23.7 \mathrm{~nm}$ rms Surface Error & $6.4 \mathrm{~nm} \mathrm{~ms}$ & $75 \mathrm{~mm} \mathrm{rms}$ \\
\hline PMSA-150: Uncorrectable Surface Error & Design to O-30 Be PEL & ULE PEL & I-70 Be PEL \\
\hline PMSA-195: Surface Change from Creep & $<10 \mathrm{~nm}$ pv sag & None & None \\
\hline PMSA 1560: ROC Adjustment Resolution & $<10 \mathrm{~nm}$ step Actuators at 30K & None & None \\
\hline PMSA 370: Hexapod 6 DOF & Operates 28-50K & $300 \mathrm{~K}$ & $4.5 \mathrm{~K}$ \\
\hline PMSA-530: Operational Temperature 28-50K & &
\end{tabular}

While there are literally 100 's of engineering specifications necessary to manufacture a JWST PMSA, only a select few are considered technology requiring demonstration:

- Gold Coating Cryo-Survivability

- Figure Thermal Stability

- Areal Density

- Figure Launch Distortion

- Primary Mirror Optical Area

- Surface Figure Error (including ROC, Hexapod, Creep \& Polishing Error)

- : Cryogenic Performance

Please note that this list of technologies is not in a priority order, but in the order of their flow down from the JWST Level 1 Science requirements developed in Tables 2 \& 3 . The balance of this section details the system engineering logic of how each mirror technology requirement flows from its originating Level 1 science requirement.

Although observatory operating temperature is a separated key technology it is really a JWST existence principle. It is the one requirement which pervades and even drives all the other requirements. To achieve the Level 1 science requirement of providing a thermal environment that permits the science instruments to have Zodiacal light background limited imaging performance (BLIP) over the wavelength range from 1.7 to 10 micrometers, the observatory must limit its thermal emissions by operating at a cryogenic temperature of less than $50 \mathrm{~K}$. This requirement directly drives the need to place the telescope at L2 which requires an EELV launch which constrains low areal density mirror segments. This requirement also directly drives all operational thermal requirements including performance, survival and stability. Thermal modeling indicates that some of PMSAs may be as cold at $28 \mathrm{~K}$.

Gold coating cryogenic survivability is a relatively minor TRL-6 technology. Level 1 Science Requirements specify a spectral range for JWST of 0.6 to 27 micrometers. This requirement, in combination with the sensitivity requirement, flows into a Level 2 optical transmission requirement which directly flows into a PMSA reflectivity requirement. Uncoated polished Beryllium (or glass) cannot achieve the required reflectivity over the required spectral range. Gold is the best candidate coating material. It provides excellent reflectivity in the near- and mid-infrared and acceptable performance in the visible. Silver does provide better performance in the visible, but it requires a protective layer to avoid oxidation problems. Aluminum, while common for ground based visible telescopes, does not have acceptable infrared performance for JWST. Gold is a common coating material and thus is not itself a TRL-6 Technology. But, the cryogenic survival of a gold coating applied to a large 0-30 Beryllium mirror has never before been demonstrated.

PMSA surface figure thermal stability is possibly the most important TRL-6 technology. This importance was reflected by it pivotal role in selecting Beryllium as the primary mirror material (JWST-RVW-002463; Stahl, 2004). Level 1 specifies that science observations must be able to occur at any position in the celestial sphere. This requirement places a stability requirement on the observatory encircled energy (EE) as it slews between celestial sphere positions - which in practice is a constraint on how much the observatory point spread function (PSF) can change its shape as a function of the thermal gradient introduced into the observatory at each celestial sphere location. At the PMSA level, EE thermal stability is directly determined by the thermal stability of the surface figure shape. While dozens of engineering issues contribute to this stability (such as material coefficient of thermal expansion (CTE) uniformity; structural design, including actuator athermalization bracket design and bi-metallic effects; etc.) it is the system level PMSA performance which is the TRL-6 technology. It is a specific PMSA design implementation which must be demonstrated to have cryogenic figure stability of less than $0.3 \mathrm{~nm} \mathrm{rms}$ per $\mathrm{K}$ of a maximum surface figure change or $7.5 \mathrm{~nm} \mathrm{rms}$ over the temperature range of 30 to $55 \mathrm{~K}$.

PMSA areal density was one of the two key technologies identified at the start of the JWST program which required significant development effort. The Level 1 science requirement of operating the observatory at L2 flowed down to a Level 2 requirement that the observatory must be launched via an EELV (such as an Areianespace Ariane 5). 
This places a maximum mass constraint of $6,159 \mathrm{~kg}$ on the observatory. The original primary mirror allocation of this mass was approximately $1000 \mathrm{~kg}$. And, given that the original telescope collecting area was to be 50 square meters, this placed an areal density requirement upon the primary mirror of approximately $20 \mathrm{~kg} / \mathrm{m} 2$. To provide margin, a technology goal of $15 \mathrm{~kg} / \mathrm{m} 2$ was defined. And, it was this goal which completely drove the entire JWST mirror technology development program. As the observatory architecture evolved and mass maturity of different observatory elements improved, the PMSA areal density specification settled at $26.5 \mathrm{~kg} / \mathrm{m} 2$.

PMSA diameter is the second key technology identified at the start of the JWST program requiring significant development effort. Originally, an 8 meter class primary mirror was required to achieve the desired observatory sensitivity. Given that the observatory would be launched to L2 on an EELV and that the maximum available EELV shroud diameter is only 4.5 meters, it was clear that a segmented and deployed architecture was required. Competing design solutions required segments with diameters ranging from 1 to 3 meters. Ground based telescopes (Keck, Hobby-Eberly) and test beds (LAMP, ALOT, AOSD) had demonstrated the ability to fabricate segments in such sizes, but their areal densities were too high (70 to $2000 \mathrm{~kg} / \mathrm{m} 2$ ) to be launched. Thus, a primary focus of the mirror technology development effort was on how to manufacture 1 to 3 meter class mirror systems with the required areal density. A key task was: to design and demonstrate a substrate which could be manufactured, safely handled, optically finished including ground testing, and integrated into a system that would survive launch - all for less than $20 \mathrm{~kg} / \mathrm{m} 2$. A side issue was the ability to manufacture the substrate blank. Pre-JWST all large mirrors were glass, which, while acceptable for ambient operating conditions, were less than ideal for a cryogenic telescope. And, the largest pre-JWST Beryllium mirror was the 0.85 meter ITTT primary mirror. Hence, a key element of the AMSD program was demonstrating the ability to manufacture a 1.5 meter class Beryllium mirror blank - as well as the entire mirror system. The current PMSA diameter requirement is derived from a combination of the Level 1 science requirement to have a minimum of 25 square meters of unobscured optical collecting area and the prime contractor's choice of an 18 segment architecture for achieving that area. The current mirror diameter requirement is slightly larger than what was demonstrated on AMSD. If instead of an 18 segment solution, a 36 segment architecture had been selected, then the required mirrors would have been slightly smaller than what was demonstrated on AMSD.

PMSA cryogenic surface figure, creep, launch distortion and adjustability requirements are derived performance metrics directly traceable to the Level 1 science requirement that the observatory shall be diffraction limited at 2 micrometers. To achieve the Level 1 requirement, the optical telescope element (OTE) is required to have a wavefront error of less than $131 \mathrm{~nm} \mathrm{rms.} \mathrm{Detailed} \mathrm{error} \mathrm{budgeting} \mathrm{by} \mathrm{Ball}$ Aerospace has partitioned this wavefront error between multiple sources, including: uncorrectable residual PMSA surface figure error; errors in the ability to adjust all PMSA's to a common radius of curvature (ROC); errors in the ability to phase all PMSA's into a common primary mirror by correcting PMSA rigid body errors; creep of a PMSA figure as a function of time; and figure change experienced by a PMSA as a function of the launch environment. Allocating performance between these capabilities involved detailed system engineering to balance a multi-dimensional trade space. The result of this process is that uncorrectable PMSA cryogenic surface figure error i.e. errors which cannot be corrected by ROC adjustment or sliding the PMSA in 'parent' space with the hexapod - must be less than or equal to $23.7 \mathrm{~nm}$ rms at delivery to the OTE integration and test (I\&T) process. And, from the time that a PMSA is delivered for OTE I\&T through EOL, its uncorrected surface figure error induced from material creep shall be less than or equal to $1.8 \mathrm{~nm}$ rms. Furthermore, the PMSA uncorrectable surface figure distortion due to launch shall be less than or equal to $2.9 \mathrm{~nm} \mathrm{rms}$. To 'fix' correctable figure errors on-orbit, each PMSA shall have the ability (at temperatures $<50 \mathrm{~K}$ ) to adjust its ROC and rigid body position. Each PMSA must be able to adjust its ROC with a resolution of less than or equal to $10 \mathrm{~nm}$ pv sag. And, each PMSA must be able to adjust is piston position with a resolution of less than or equal to $10 \mathrm{~nm}$.

An interesting sub-element of these requirements is the role of material stress/strain and precision elastic limit (PEL) on PMSA design and its interconnections with figure creep, launch deformation, surface figure error and areal density. To meet the creep and launch figure change requirements, it is critical that the PMSA substrate is designed with sufficient stiffness to avoid introducing excessive stress/strain into the mirror during the optical fabrication process. It is the release of this stress/strain from the mirror with time or exposure to the launch environment (vibration and acoustic) which causes undesired figure change. PMSA stiffness is also important for in-process optical testing and OTE I\&T, the mirror must have sufficiently small gravity sag that it can be accurately measured in one-g (i.e. on the Earth) while being manufactured for optimized performance in zero-g. So, while AMSD demonstrated that a PMSA with areal density $<20 \mathrm{~kg} / \mathrm{m} 2$ is achievable, a PMSA areal density specification of $26.5 \mathrm{~kg} / \mathrm{m} 2$ is necessary to produce a PMSA with sufficient stiffness to meet the other requirements. 


\section{Requirements Verification}

All PMSA technologies necessary to meet the JWST Level 1 requirements have been demonstrated to be at TRL-6. As described in the below paragraphs, this was accomplished via a systematic mirror technology demonstration program (SBMD, AMSD and JWST) to mature enabling mirror design and fabrication technologies for JWST. As a precursor to AMSD, SBMD matured specific separable technologies and demonstrated their performance in a relevant environment, for example, gold coating performance at $28 \mathrm{~K}$. AMSD was critical for certifying TRL-6 compliance. AMSD produced a complete mirror system (with a design that is traceable to flight) and tested its performance in a relevant environment from 30 to $50 \mathrm{~K}$.
While AMSD was designed to explore fabrication limits associated with areal density and size, it could not certify everything. Some technologies had to wait for testing with actual JWST flight segments. For example, AMSD was not designed (nor ever intended) to meet JWST launch loads. To survive launch, JWST flight PMSAs are designed to have significantly more areal density than AMSD (which actually makes a JWST PMSA easier to fabricate). Hence, it was necessary to use an actual JWST flight PMSA for vibration and acoustic testing. To aid in determining when TRL-6 was achieved for PMSA mirror technology, specific success criteria were established for each critical technology based upon the Equipment Specification for JWST PMSA (Ball, 2005). Compliance of PMSA mirror technology with these criteria was verified by test (Table 4).

\begin{tabular}{|c|c|c|c|}
\hline \multicolumn{4}{|c|}{ Table 4 Mirror Technology Success Criteria } \\
\hline PMSA Technology & Success Criteria & Achieved & Method \\
\hline PMSA-110: Spectral Reflectance $0.6-28 \mu \mathrm{m}$ & Gold Coating on $\mathrm{O}-30 \mathrm{Be}$ & Gold Coating on $\mathrm{O}-30 \mathrm{Be}$ & SBMD \\
\hline PMSA-530: Operational Temperature $28-50 \mathrm{~K}$ & with $28 \mathrm{~K}$ Survival & with $28 \mathrm{~K}$ Survival & \\
\hline PMSA-170: Surface Figure Thermal Change & $<7.5 \mathrm{nmms}$ for 30 to $55 \mathrm{~K}$ & $7 \mathrm{~nm} \mathrm{rms} \mathrm{from} 30$ to $55 \mathrm{~K}$ & $\overline{\text { AMSD }}$ \\
\hline PMSA-410: Mass < $39.17 \mathrm{~kg}$ & Areal Density $<26.5 \mathrm{~kg} / \mathrm{m} 2$ & Areal Density $=15.6 \mathrm{~kg} / \mathrm{m} 2$ & $\begin{array}{l}\text { AMSD } \\
\text { JWST B1 }\end{array}$ \\
\hline $\begin{array}{l}\text { PMSA-180: Surface Distortion from Launch } \\
<2.9 \mathrm{~nm} \text { rms }\end{array}$ & $\begin{array}{l}\text { Less than metrology error } \\
\text { budget of } 14 \mathrm{~nm} \mathrm{rms}\end{array}$ & $\begin{array}{l}10.6 \mathrm{nmm} \text { rms Surface Change } \\
\text { from Vib \& Acoustic Test }\end{array}$ & JWST B1 \\
\hline PMSA-70: Polished Surface Area $>1.46 \mathrm{~m} 2$ & $\begin{array}{l}1.3 \text { meter diameter Segment } \\
\text { delivered from AXSYS }\end{array}$ & $\begin{array}{l}1.3 \text { meter diameter Segment: } \\
\text { delivered from AXSYS }\end{array}$ & $\begin{array}{l}\text { AMSD } \\
\text { JWST }\end{array}$ \\
\hline PMSA-150: Uncorrectable Surface Error & $<23.7 \mathrm{~nm} \mathrm{rms}$ Surface Error & $\begin{array}{l}18.8 \mathrm{~nm} \mathrm{rms} 30 \mathrm{~K} \text { Figure } \\
19.2 \mathrm{~nm} \mathrm{~ms} 300 \mathrm{~K} \text { Figure }\end{array}$ & $\begin{array}{l}\text { SBMD } \\
\text { AMSD }\end{array}$ \\
\hline $\begin{array}{l}\text { PMSA-195: Surface Change from Creep } \\
<1.8 \mathrm{~nm} \mathrm{~ms}\end{array}$ & Design to O-30 Be PEL. & $\begin{array}{l}\text { Designed to ensure }<2000 \mathrm{psi} \\
\text { residual stress }\end{array}$ & $\begin{array}{l}\text { SBMD } \\
\text { AMSD } \\
\text { JWST }\end{array}$ \\
\hline PMSA 1560: ROC Adjustment Resolution & $<10$ nmpv sag & $0.8 \mathrm{~nm}$ pv sag & AMSD \\
\hline PMSA 370: Hexapod 6 DOF & $<10 \mathrm{~nm}$ step Actuators at $30 \mathrm{~K}$ & $7.5 \mathrm{~nm}$ step Actuators at $30 \mathrm{~K}$ & $\begin{array}{l}\text { AMSD } \\
\text { JWST }\end{array}$ \\
\hline PMSA-530: Operational Temperature $28-50 \mathrm{~K}$ & Operates $28-50 \mathrm{~K}$ & Operated at $28-50 \mathrm{~K}$ & AMSD \\
\hline
\end{tabular}

The maturity of mirror technology to satisfy the (derived from PMSA requirement PMSA-110 \& PMSA-530) ability of a gold coating (that provides the necessary spectral transmission properties when deposited on to an O-30 beryllium mirror) to survive $28 \mathrm{~K}$ temperatures was verified with SBMD. TRL-6 was demonstrated by performance testing at $30 \mathrm{~K}$ and survival testing to $28 \mathrm{~K}$ a gold coating deposited on to the SBMD 0.5 meter O-30 beryllium mirror. Since cryogenic adhesion of gold on $\mathrm{O}-30$ beryllium was the primary ability being tested and not the ability to deposit gold coatings on to large mirrors, it was determined that repeating the test with a gold coated AMSD mirror was unnecessary. The deposited gold coating introduced no discernible cryogenic surface figure distortion into SBMD. The uncoated SBMD's $30 \mathrm{~K}$ surface figure was $52.8 \mathrm{~nm} \mathrm{rms}$ and its coated $30 \mathrm{~K}$ surface figure was $53.9 \mathrm{~nm} \mathrm{rms.} \mathrm{(Fig} \mathrm{3)}$ (Reed and Kendrick, 2000)
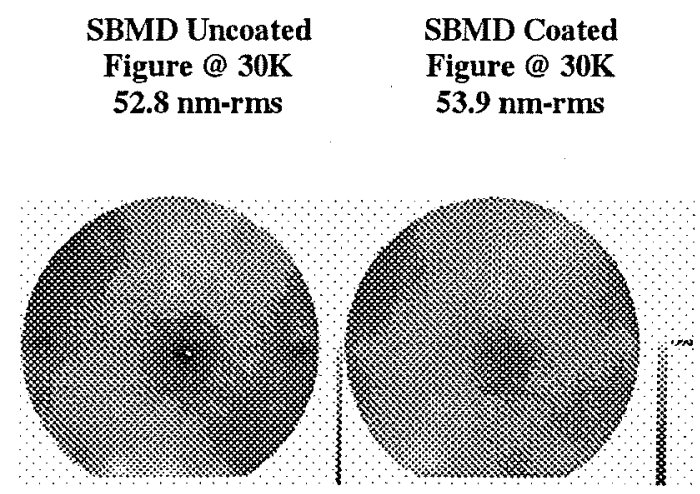

Figure 3 Results of SBMD BATC-IRAD Coating Demonstration 
The maturity of mirror technology to satisfy the balance of PMSA requirement PMSA-530, ability to operate over a 28 to $50 \mathrm{~K}$ temperature range, was verified with AMSD and JWST flight actuators. TRL- 6 was demonstrated by testing the AMSD beryllium mirror system multiple times over operational temperatures from 28 to $50 \mathrm{~K}$ to characterize its cryogenic performance. Cryogenic figure stability was characterized. Cryogenic figure and radius of curvature change was demonstrated. And, cryogenic radius of curvature adjustability was demonstrated. TRL-6 was further demonstrated by testing the cryogenic performance of JWST flight actuators.

The maturity of mirror technology to satisfy PMSA requirement PMSA-170, ability of a PMSA to maintain a surface figure stability of less than $0.3 \mathrm{~nm}$ rms for a $1 \mathrm{~K}$ temperature change $(7.5 \mathrm{~nm} \mathrm{rms}$ over a 30 to $55 \mathrm{~K}$ thermal range), was verified with AMSD. TRL-6 was demonstrated by measuring the surface shape of the AMSD beryllium mirror system as a function of temperature. The cryogenic surface figure was measured at multiple temperatures. The surface figure was found to change linearly with temperature. The total surface figure change from 30 to $55 \mathrm{~K}$ was $7.0 \mathrm{~nm}$ rms or $0.28 \mathrm{~nm} \mathrm{rms}$ per $1 \mathrm{~K}$ temperature change. (Figure 4) (JWST-RVW-002463; Stahl, 2004)

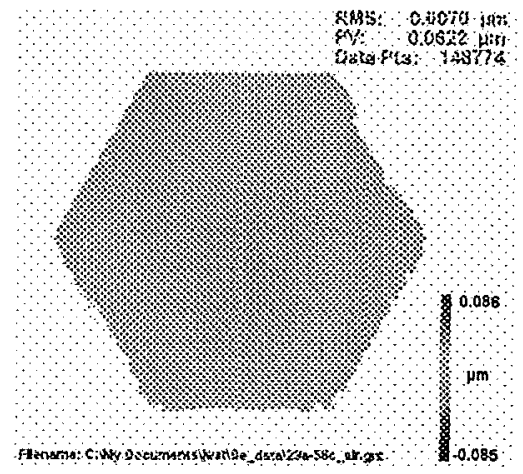

Figure 4 Measured Cryogenic

Figure Stability from 30 to 55

The maturity of mirror technology to satisfy the (derived from PMSA requirement PMSA-410 and PMSA-70) ability to manufacture a PMSA with areal density less than 26.5 $\mathrm{kg} / \mathrm{m} 2$ was verified with AMSD and confirmed with JWST flight segments. TRL- 6 was demonstrated by calculating the areal density of the AMSD beryllium mirror segment and of an assembled JWST PMSA B-1 (Figure 5) from measurements of their respective masses and physical dimensions. The achieved areal density for JWST PMSA
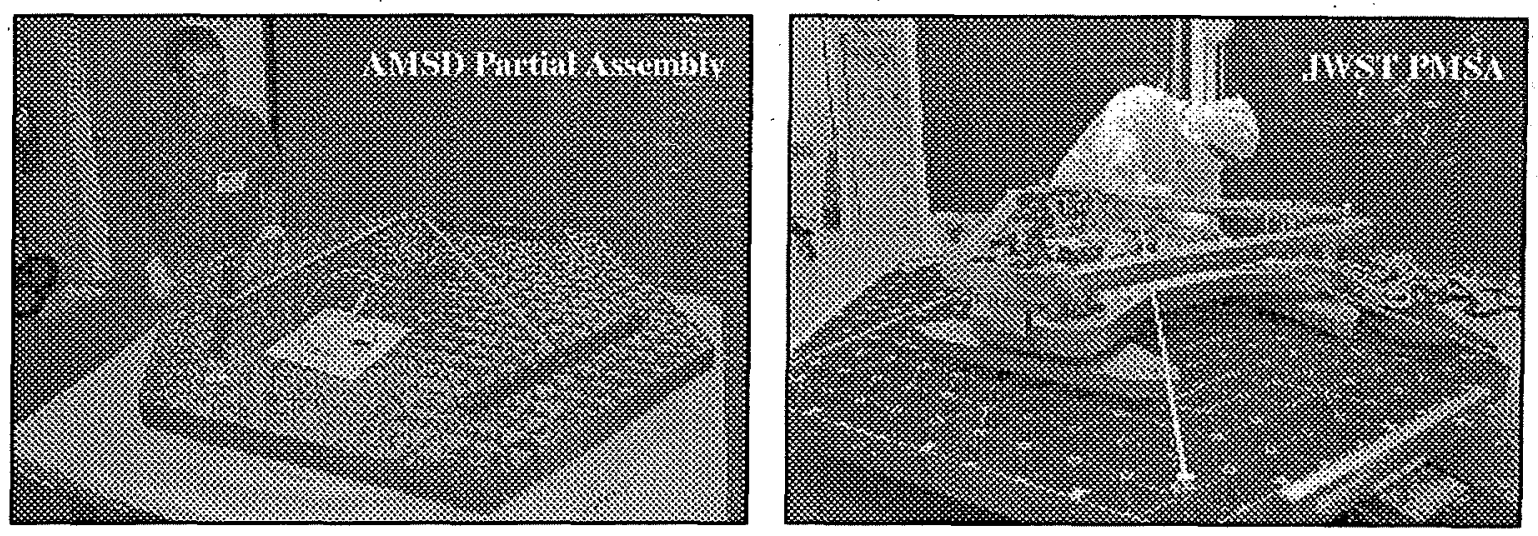

Figure 5 Assembled AMSD and JWST PMSA mirror systems

B-1 is $25.8 \mathrm{~kg} / \mathrm{m} 2$. AMSD actually demonstrated the feasibility of manufacturing a mirror system with an areal density of $15.6 \mathrm{~kg} / \mathrm{m} 2$. This was achieved by CNC machining a beryllium mirror substrate with exceptionally thin ribs and facesheet while controlling the introduction of residual stress. Residual stress is very important. It can adversely affect the ability to polish a beryllium mirror to the required surface figure and keep that shape because of long term figure creep. The higher JWST PMSA areal density requirement allowed for design maturity, incorporating lessons learned from the AMSD project and validated with improved modeling, to ease manufacturability and reduce risk.

The maturity of mirror technology to satisfy PMSA requirement PMSA-70, ability to manufacture a PMSA with a polished surface area of larger than 1.46 square meters, was verified with a combination of SBMD, AMSD and JWST. TRL-6 is verified with three specific demonstrations of fact. First, the JWST flight program is successfully manufacturing $18 \quad 1.315$ meter flat to flat beryllium substrates. And, at present, flight mirrors are in various stages of surface grind and polish. While this may seem trivial now, before the JWST mirror technology development program, there was great uncertainty as to whether or not the manufacture of beryllium substrates of that size was even feasible. Second, AMSD demonstrated the ability to fabricate a 1.2 meter flat to flat polished beryllium mirror with a mechanical design and aspheric prescription traceable to JWST. Until it is surpassed by JWST, AMSD is the largest diameter beryllium mirror ever fabricated. Third, SBMD demonstrated the ability to use small tool polishing of a lightweight mirror substrate to within $5 \mathrm{~mm}$ of a straight edge. 
The maturity of mirror technology to satisfy PMSA requirement PMSA-150, ability to polish a PMSA with an uncorrectable surface figure error of less than $23.7 \mathrm{~nm} \mathrm{rms}$, was verified with SBMD and AMSD. TRL-6 was confirmed by verifying two key abilities: 1) ability to polish a large-aperture low-areal-density aspheric $\mathrm{O}-30$ beryllium mirror to the required specification and 2) ability to cryonull figure an $0-30$ beryllium mirror to have the required figure specification at temperatures $<50 \mathrm{~K}$. The ability to polish a meter-class highly-aspheric lightweight O-30 beryllium mirror was demonstrated on AMSD. AMSD was polished to have an uncorrectable surface figure error of 19.2 $\mathrm{nm}$ rms over 97.1 percent of its aperture (Figure 6).

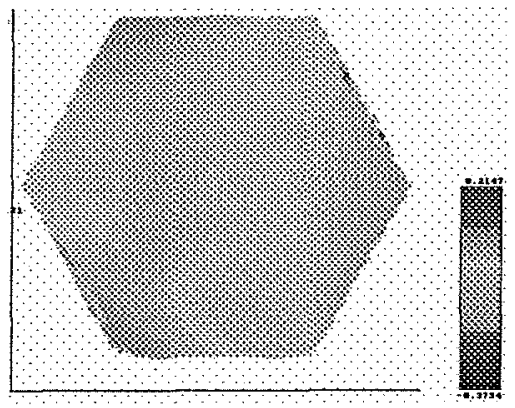

Figure 6 Final AMSD Ambient Surface Figure is $19.2 \mathrm{~nm}$ rms (97.1\% of Mirror Area)
Achieving a $<20 \mathrm{~nm}$ rms surface figure was actually the last major task of the AMSD program. And, its accomplishment represented a never before demonstrated capability for meter-class lightweight beryllium mirrors. Furthermore, because AMSD had a 10 meter radius of curvature (ROC), it was a more difficult prescription to polish than JWST segments with their 16 meter ROC. The $<20 \mathrm{~nm}$ rms uncorrectable surface figure was achieved via a small tool computer controlled optical surfacing (CCOS) technology at Tinsley Laboratories in Richmond CA. Critical to this accomplishment was high spatial sampled data and precision fiducial registration knowledge. The ability to cryo-null figure such a mirror to yield the required surface figure error at cryogenic temperatures was demonstrated on SBMD. SBMD exhibited a cryodeformation of approximately $90 \mathrm{~nm} \mathrm{rms}$. This shape changed consisted of a low-order mount induced error and a high-order quilting error associated with the substrate rib structure. After two cryo-cycles "proved that the deformation was stable and repeatable, i.e. that the 0-30 beryllium mirror had no apparent creep induced figure change associated with residual stress in the mirror, SBMD was cryo-null figured. SBMD was cryo-null figured using Tinsley small tool CCOS technology to correct both low \& high-order errors. The predicted final cryogenic surface figure was $14.4 \mathrm{~nm}$ rms. The actual final cryogenic surface error was $18.8 \mathrm{~nm} \mathrm{rms} \mathrm{(Fig} \mathrm{7).} \mathrm{(Reed} \mathrm{and} \mathrm{Kendrick,} \mathrm{2000)}$

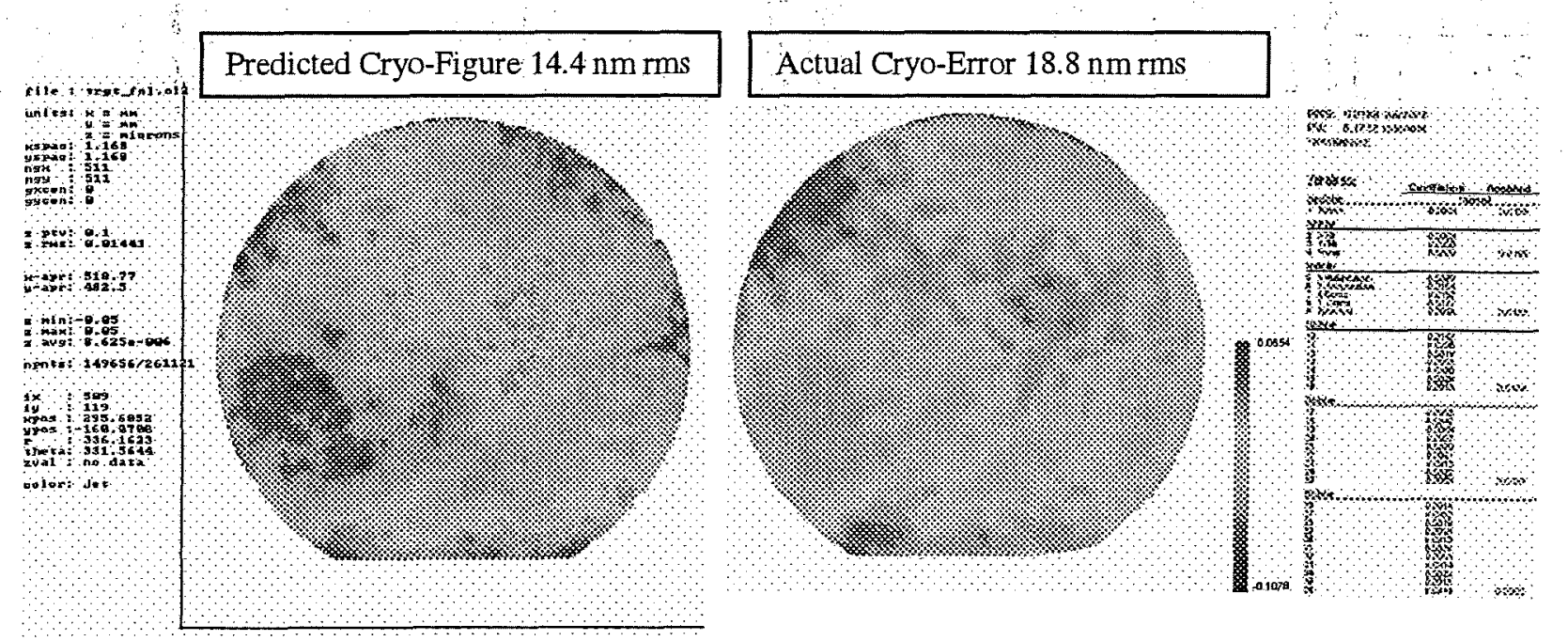

Figure 7 SBMD Predicted Cryo-Figure of $14.4 \mathrm{~nm}$ rms vs Actual Cryo-Null Figured Cryogenic Surface Error of $18.8 \mathrm{~nm}$

Based upon the SBMD success of cryo-null figuring via small tool CCOS technology both low-order mount induced as well as high-order rib structure quilting, it was determined un-necessary to cryo-null figure AMSD.

The maturity of mirror technology to satisfy PMSA requirement PMSA-370, ability to position a PMSA in space with 6 degrees of freedom (DOF) with less than 10 $\mathrm{nm}$ step resolution, was verified with AMSD and JWST engineering unit components. TRL- 6 was demonstrated by test of the JWST actuator performance at $30 \mathrm{~K}$ and analysis of JWST hexapod motion at $30 \mathrm{~K}$. The technology that achieves the required capability is a cryogenic hexapod mechanism with six cryogenic actuators which controls the $6 \mathrm{DOF}$ position of a mirror segment relative to the JWST telescope backing structure. A $7^{\text {th }}$ actuator is used to deflect the center of the mirror, changing the radius of curvature for that segment. While the use of a hexapod is not new technology, the actuator step size resolution required at cryogenic temperature is. To meet the hexapod motion resolution and accuracy requirements, the JWST actuators 
must be independently capable of less than $10 \mathrm{~nm}$ step size resolution at $<50 \mathrm{~K}$. This level of motion resolution is achieved when the JWST actuators are operated in their 'fine' mode. JWST actuators are actually dual stage with coarse and fine operating modes. The JWST actuators were developed by BATC, initially under IRAD funding, and then via AMSD, to meet specific mass, stiffness, and performance requirements. These actuators are used for both PMSA hexapod and radius of curvature adjustments. The key component of the JWST actuator is a cryogenic capable geared stepper motor which was derived from the gear motor flown on the Spitzer Space Telescope and operated at $4.5 \mathrm{~K}$. TRL- 6 capability has been demonstrated by characterizing cryogenic performance from 25 to $35 \mathrm{~K}$ of (to date) 24 JWST actuators: 2 actuators via Ball $\mathrm{IRAD}, 4$ actuators via AMSD (Kingsbury, 2002) and 18 JWST engineering unit actuators. All actuators have met the resolution requirement with the JWST engineering unit actuators showing a resolution of $7 \mathrm{~nm}$ (Figure 8).

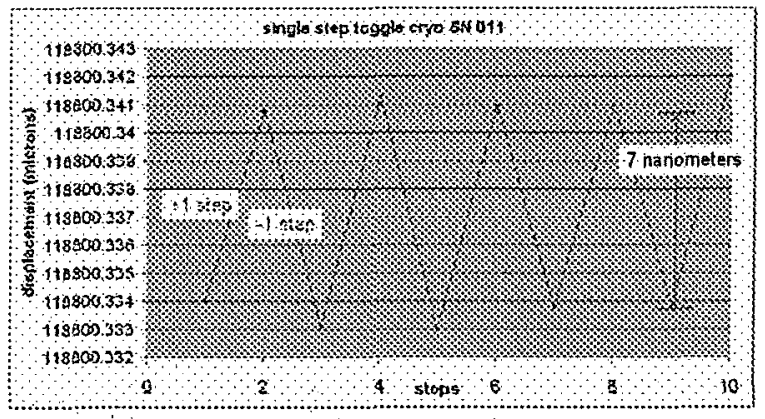

Figure 8 Actuator Single Step Resolution at $25 \mathrm{~K}$

Extensive testing of the actuators through a variety of fine stage step increments verified that the actuator performs single step moves, without backlash, to an accuracy of 0.6 $\mathrm{nm} \mathrm{rms}$. Finally, flight actuators have been installed into a flight hexapod system and exercised at ambient temperature to show basic functionality.

The maturity of mirror technology to satisfy PMSA requirement PMSA-1560, ability to adjust a PMSA cryogenic radius of curvature (ROC) sag by less than $10 \mathrm{~nm}$ peak-to-valley (pv), was verified with AMSD. TRL-6 was demonstrated by test, analysis and corollary. JWST PMSA mirrors are designed to allow their radius of curvature to be adjusted at cryogenic temperatures by expanding or contracting a linear actuator. The actuator, attached to the back center of the mirror, re-acts its force via six struts that interface to each mirror corner through a flexured joint. A similar design was implemented on AMSD except the actuator re-acted its force against spreader bars. (Figure 5) While at $30 \mathrm{~K}$, the AMSD actuator was commanded to execute 'coarse-steps' until a detectable ROC sag change was measured. A total move of 35 coarse steps resulted in a ROC sag change of $38 \mathrm{~nm}$ pv. By analysis, a single AMSD 'coarse-step' should result in a ROC sag change of approximately $1.1 \mathrm{~nm}$ pv. And, a single 'fine-step' motion (which is 4.5 times smaller than a coarse step) should result in a ROC sag change of approximately $0.24 \mathrm{~nm}$ pv. (JWSTRVW-002463) Because of difference between where the actuator force re-acts against the mirror substrate, the distance between those re-action points and the intrinsic stiffness between AMSD and JWST, a JWST PMSA experiences a ROC sag change that is approximately $110 \%$ larger per linear motion than AMSD experiences. Thus, the minimum JWST coarse step is approximately $1.2 \mathrm{~nm}$ pv and the minimum fine step is approximately $0.27 \mathrm{~nm} \mathrm{pv}$.

The maturity of mirror technology to satisfy PMSA requirement PMSA-195, ability to design a PMSA whose surface figure changes by less than $1.8 \mathrm{~nm}$ rms because of creep, was verified with SBMD, AMSD and JWST flight segments. TRL- 6 was demonstrated by test and analysis. Funded via AMSD, Draper Laboratory measured the creep properties of $0-30$ beryllium (Mustone, 2002). Significant creep was measured for samples stressed to 4 and $6 \mathrm{ksi}$. Neglible creep was measured for samples stressed to $2 \mathrm{ksi}$ or below (Figure 9).

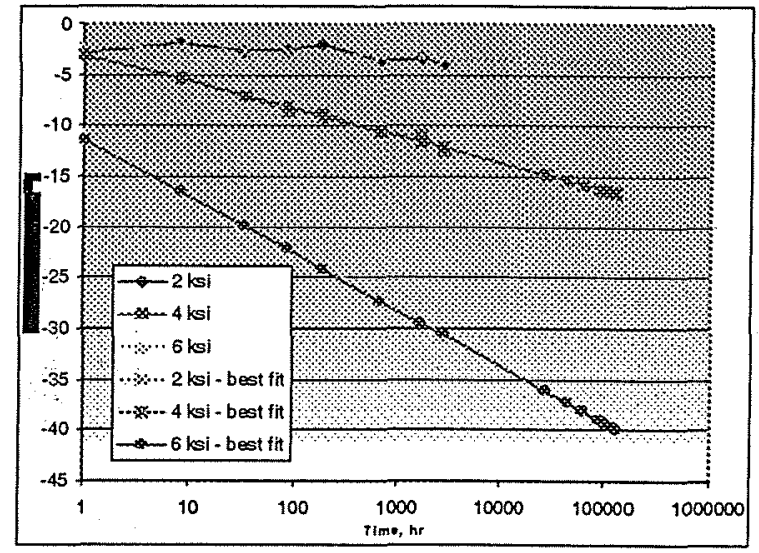

Figure 9 Draper Labs Creep Test Data

Analysis indicates that $2 \mathrm{ksi}$ of stress will creep 1.8 parts per million over 10 yrs at room temperature (Kradinov, 2004). Further analysis indicates that a PMSA with a surface stress of $2 \mathrm{ksi}$ will see a total figure change of less than $1.8 \mathrm{~nm}$ rms during its room temperature life prior to launch. And, that no figure change due to creep is expected on orbit at cryogenic temperatures. A rule was established that all beryllium components of the JWST PMSA must be designed, processed and handled in such a way that no component has a residual stress of greater than $2 \mathrm{ksi}$. Additionally, extensive tests were performed under AMSD III to quantify exactly how much stress is introduced into a Be mirror during the machining process at AXSYS and grinding/polishing process at Tinsley. These processes are controlled to limit residual stress in the final mirrors to less than $2 \mathrm{ksi}$. Furthermore, all Be components are stress relieved throughout the fabrication process to prevent the accumulation of stress.

The maturity of mirror technology to satisfy PMSA requirement PMSA-180, ability of a PMSA to survive 
launch with less than $2.9 \mathrm{~nm} \mathrm{rms}$ surface figure distortion, was verified with a JWST flight segment. TRL6 was demonstrated by test. An unpolished JWST mirror segment B1 was assembled into a flight configuration PMSA and exposed to design limit loads with sine burst, random vibration, and acoustic testing. Its surface figure change as a function of each loading test was measured using a Phase Measuring Electronic Speckle Pattern Interferometer (ESPI). The design limit load accelerations for every component within the PMSA were exceeded in each of these tests. Two acoustic tests were performed. The first test hard mounted the PMSA to a concrete wall. The second test suspended the PMSA for a "free-free" test. (Figure 10) The two different boundary conditions provide valuable information for finalizing the PMSA test environment. Neither test resulted in any measurable change to the PMSA surface figure.

The tests showed that the surface figure was repeatable to within the noise floor of the metrology system, $14 \mathrm{~nm} \mathrm{rms}$. (Figure 11, 12, 13) Astigmatism and Power figure terms are removed from the total surface change measurement because they can be compensated for on-orbit by adjusting the PMSA radius of curvature or physical location via the cryogenic hexapod with ROC actuator. Measurement of a surface figure error change that is smaller than the measurement noise floor is consistent with the pre-test prediction. Non-linear plastic material finite element analysis predicted a surface figure deformation of only 1.6 nm rms.

While not effecting a determination of demonstrating TRL6 , there were two special circumstances associated with this test. First, the random vibration and acoustic levels were notched to maintain safe exposure levels on the PMSA. And, a minor inconsistency was discovered with the design limit loads. A new test environment will be defined and a minimal PMSA redesign is anticipated to meet the new test environment. Once the modified PMSA is retested it will meet TRL8. Second, while all flight PMSAs will be thermally cycled to $25 \mathrm{~K}$ before launch, for reasons of expedience and convenience, the B1 PMSA was only thermally cycled to $150 \mathrm{~K}$. This was determined acceptable because the $150 \mathrm{~K}$ temperature subjected the mirror to $88 \%$ of the beryllium cryo strain and over $70 \%$ of adhesive mount strain. Additionally, an extensive qualification program was conducted for the bonded joints. Test samples were cycled three times between $15 \mathrm{~K}$ and $383 \mathrm{~K}$ and subjected to static pull testing. These samples saw only a $12 \%$ reduction in ultimate strength following thermal cycling and still maintained a margin of safety of 7.4. This testing coupled with the $150 \mathrm{~K}$ the $\mathrm{B} 1$ segment saw is more than sufficient to assure that the TRL6 vibration testing demonstrated the true robustness of the PMSA. TRL6 has been achieved by demonstrating the technology to design a lightweight beryllium mirror to design limit loads, testing it to those loads, and showing surface figure stability after exposure to the design limit load. Thus assuring that lightweight beryllium mirror technology can meet the JWST launch distortion requirements.

\subsection{SUMMARY}

Since 1996, all key mirror technology for a JWST Primary Mirror Segment Assembly (PMSA), as defined directly from the JWST Level 1 Science Requirements, has been developed and matured from a Technology Readiness Level (TRL) of 3 to 6 . This has occurred as the result of a highly successful technology development program including the Sub-scale Beryllium Mirror. Demonstrator (SBMD) project, Advanced Mirror System Demonstrator (AMSD) project and the JWST flight mirror fabrication. Directly traceable prototypes (and in some cases the flight hardware itself) has been built, tested and operated in a relevant environment.
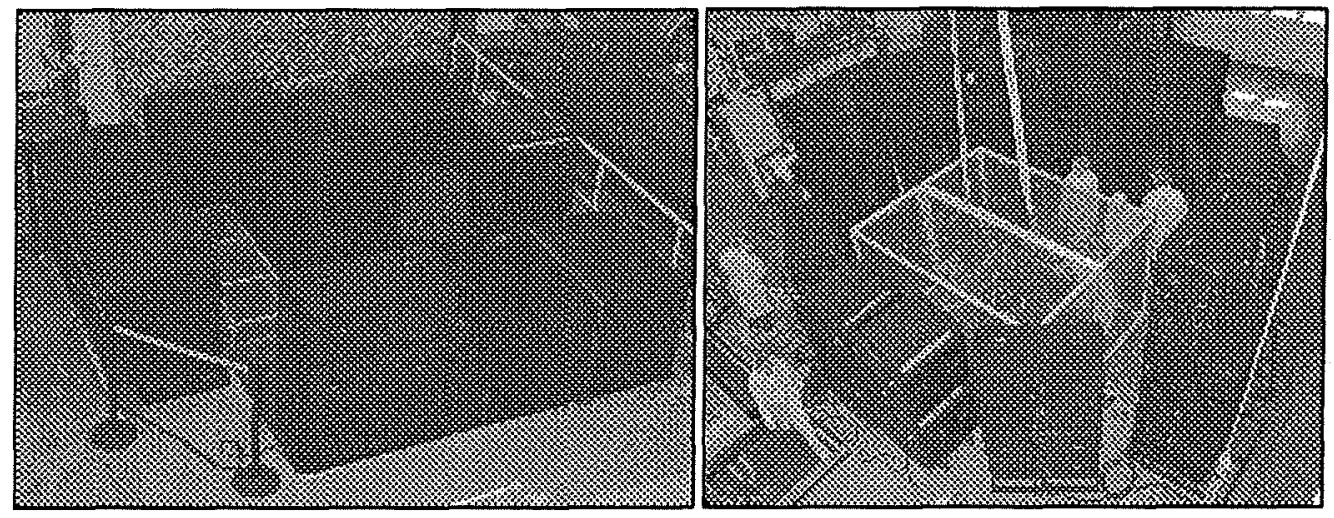

Figure 10 PMSA during first and second acoustic tests 


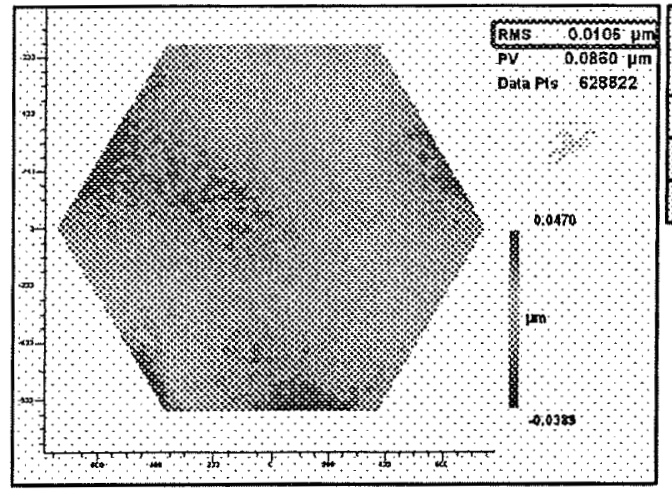

\begin{tabular}{|c|c|c|}
\hline & unplos & Orotology \\
\hline flour & 98 & 14 \\
\hline As Usmatsin & 42 & 10 \\
\hline Pown & 1115 & 70 \\
\hline
\end{tabular}

Figure 11 Figure change from exposure to 3 axis sine burst testing to design limit loads

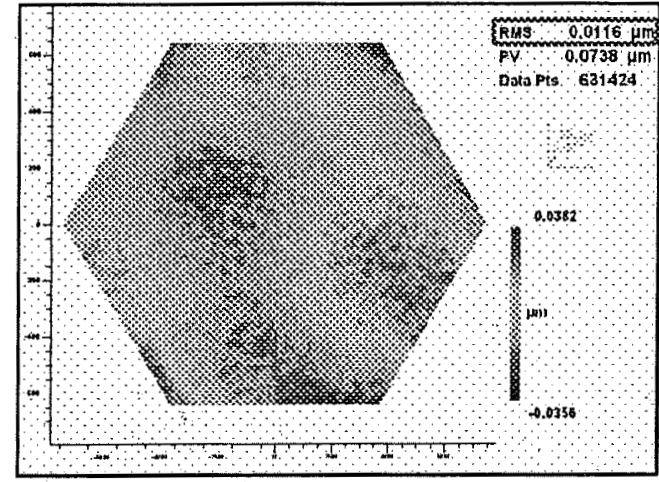

m l,

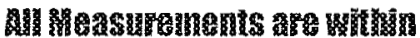

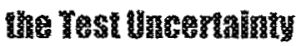

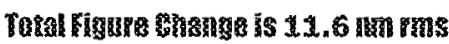

Figure 12 Figure change from exposure to 3 axis sine burst testing to design limit loads and first acoustic test

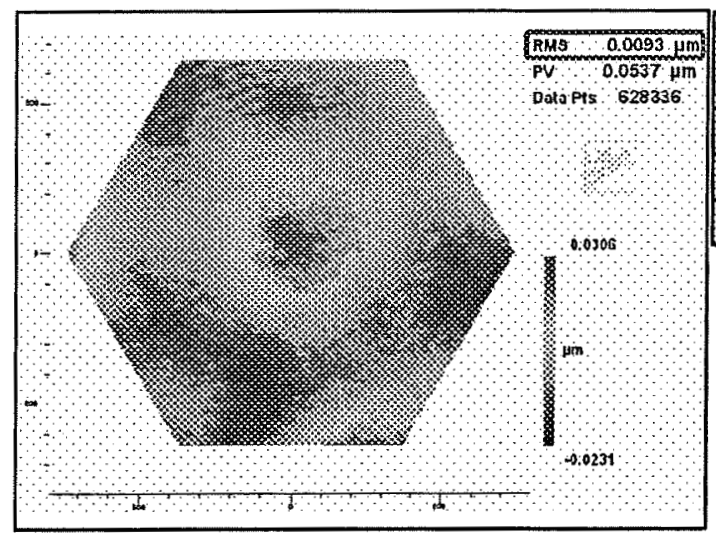

\begin{tabular}{|c|c|c|}
\hline & Mesurenent & Undoros \\
\hline floure & 93 & 14 \\
\hline $0 \mathrm{stg} \mathrm{matsm}$ & 16 & 10 \\
\hline power & 16 & 10 \\
\hline
\end{tabular}

\section{มี} แำ Tละ

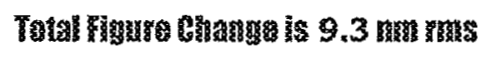

Figure 13 Figure change from exposure to second acoustic test 


\section{REFERENCES}

HST and Beyond - Exploration and the Search for Origins: A Vision for Ultraviolet-Optical-Infrared Space Astronomy, Alan Dressler, Ed., The Association of Universities for Research in Astronomy, Inc., Washington, D.C., (1996)

Next Generation Space Telescope: Visiting a Time When Galaxies Were Young, H. S. Stockman, Ed., The Association of Universities for Research in Astronomy, Inc., Washington, D.C., (1997)

Coulter, Daniel, R. "Technology development for the NextGeneration Space Telescope: an overview", Proc. SPIE Int. Soc. Opt. Eng. Vol. 3356, page 106 (1998)

NGST "Yardstick" Mission, Pierre Y. Bely, Ed., NGST Monograph No. 1 (1999)

Bely, Pierre, "Large Space Optics", NGST Science and Technology Exposition, ASP Conference Series, Eric Smith, Ed., Vol. 207, page 25 (2000)

Cox, Charles D. and Mark E. Furber, "Four-meter diameter adaptive optical system technology demonstration", Proc. SPIE Int. Soc. Opt. Eng. 2807, page 132 (1996)

Smith, W. Scott and H. Philip Stahl, "Overview of mirror technology development for large lightweight space-based optical systems", Proc. SPIE Int. Soc. Opt. Eng. Vol. 4198, page 1 (2001)

Stahl, H. Philip, "Development of lightweight mirror technology for the next generation space telescope", Proc. SPIE Int. Soc. Opt. Eng. Vol. 4451, page 1 (2001)

Reed, T. et al, "Final results of the Subscale Beryllium Mirror Demonstrator (SBMD) program", SPIE Vol.4451, pp.5, (2001)

JWST-RVW-002463, JWST Optical Telescope Element Optics Review (OOR), 9 Sept 2004.

Stahl, H. Philip, Lee D. Feinberg and Scott C. Texter, "JWST Primary Mirror Material Selection", SPIE Vol.5487, p.818, 2004.

JWST Program Plan, NASA, JWST-PLAN-000633 Rev A, (2006)

JWST Mission Requirements Document, NASA, JWSTRQMT-000634 Rev N, (2006)

Equipment Specification for the JWST Primary Mirror Segment Assembly (PMSA), BATC, EQ7-0509, (2005)
Subscale Beryllium Mirror Demonstrator (SBMD) Final Report NAS 9-98097, Timothy Reed and Stephen Kendrick, (2000)

Kingsbury, Lana, "AMSD Actuator Test Results", Systems Engineering Report SER-AMSD-011, BATC, 2002.

Mustone, Tim, "Microcreep Testing of $\mathrm{O}-30 \mathrm{H}$ Beryllium, Final Update of Data", Draper Laboratory Memo No GBB202-062-Rev 2, 9 December 2002

Kradinov, Vladimir, 'O-30H Beryllium Creep Modeling', Smithsonian Astrophysical Observatory Memo, 4 October 2004.

\section{BIOGRAPHY}

Dr. H. Philip Stahl earned his Ph.D. in Optical Sciences from the University of Arizona Optical Sciences Center in 1985. He is currently a Senior Optical Physicist at NASA MSFC where he is the JWST Optical Components Lead responsible for procurement insight/oversight for the JWST primary, secondary and tertiary mirrors; NGST Mirror Technology Lead responsible for developing candidate primary mirror

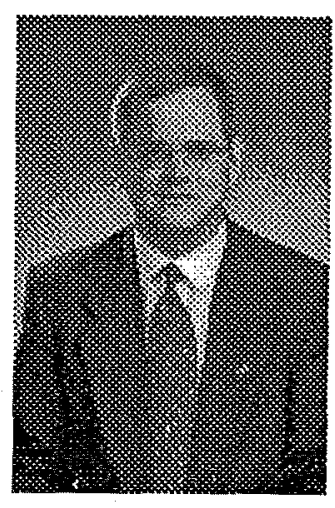
technologies; and, AMSD Technology Lead. Dr. Stahl is a leading authority in optical metrology, optical engineering, and phase-measuring interferometry. Many of the world's largest telescopes have been fabricated with the aid of highspeed and infrared phase-measuring interferometers developed by Dr. Stahl, including the Keck, VLT and Gemini telescopes.

Previously, Dr. Stahl was a Senior Staff Optical Engineer at Raytheon Danbury (formerly Hughes Danbury Optical Systems and now.Goodrich Aerospace) where he was lead optical engineer on the $4 \mathrm{~m}$ Alpha/Lamp Integration program, on the Space Based Laser Program and for $4 \mathrm{~m}$ metrology capability. As President of Stahl Optical Systems Inc. he supported two microgravity experiments: the Surface Tension Driven Convection Experiment (STDCE-2) and the Droplet Combustion Experiment (DCE). Also, he was an Assistant Professor of Physics and Applied Optics at Rose-Hulman Institute of Technology, the Optical Products Manager at Breault Research Organization.

Dr. Stahl is a member of OSA and SPIE (Fellow). He received his BA in Physics and Mathematics from Wittenberg University (1979), and his MS and Ph.D. in Optical Sciences from the University of Arizona (83 \& 85). 